\title{
Improved Imaged-derived Input Function for Study of Human Brain FDG-PET
}

\author{
Hongbin Guo, Rosemary A Renaut and Kewei Chen
}

\begin{abstract}
A reliable, semi-automated method for estimation of a minimally-invasive image-derived input function is validated for human $\left[{ }^{18} \mathrm{~F}\right]$-fluoro deoxyglucose (FDG) positron emission tomography (PET) studies. Two time windows can be recognized in the time activity curve measured from a carotid artery region of interest (CA-ROI). During the first short window of very rapid change, the dominant contamination of the measured activity on the CA-ROI is due to partial volume effects. After this the measured activity is also contaminated by spillover of tracer from tissue to blood. Blood samples acquired at later times, free of both partial volume and spillover effects, avoid the contamination and are used to fit a functional form for the input. Appropriateness of this parameter dependent input is validated using data from 18 healthy subjects and contrasted against measured arterial blood samples, and with previously proposed representations [1]-[3]. Parameters defining the input function and micro parameters of each tissue cluster, (their rate constants $K_{1}, k_{2}$ and $k_{3}$ ), [4], are estimated simultaneously using the compartmental model for FDG PET [5].
\end{abstract}

\section{Index Terms}

Quantification of FDG PET, Automated Image-derived input function, Neuroimaging.

This work was partially supported by the Arizona Center for Alzheimer's Disease Research which is funded by the Arizona Department of Health Services, by NIH grant EB 2553301 and for the third author also by the National Institute on Aging grant R01 MH57899.

Hongbin Guo and Rosemary A Renaut are with Arizona State University, Department of Mathematics and Statistics, Tempe, AZ 85287-1804. Tel: 480-965-8002, Fax: 480-965-4160. Email: hb_guo@asu.edu and renaut@asu.edu

Kewei Chen is with Positron Emission Tomography Center, Banner Good Samaritan Medical Center, Phoenix, AZ 85006. Email: Kewei.Chen@bannerhealth.com 


\section{Improved Imaged-derived Input Function for Study of Human Brain FDG-PET}

\section{INTRODUCTION}

Positron emission tomography (PET) is a widely used neuroimaging technique that, among others, assists with disease diagnosis, treatment evaluation and study of brain function. In addition to its visual and qualitative assessment, PET images can provide quantitative estimates of physiological or metabolic parameters of interest. For example dynamic $\left[{ }^{18} \mathrm{~F}\right]$-fluoro deoxyglucose (FDG) PET allows the estimation of the cerebral metabolic rate for glucose (CMRGLc), or even individual kinetic rate constants describing various aspects of the tracer biochemistry/physiology, and has been recommended as the standard for analyses of brain diseases associated with aging, [6]. For such quantification, the input function, the time-varying radiotracer concentration in plasma, is required, whether obtained invasively or using an imagederived technique.

While the invasive gold-standard arterial blood sampling procedure to obtain the input function has long been suggested, its invasive nature has prevented its routine use in basic research, especially in clinical PET studies. Many research groups have proposed alternative methods. These include, not only for FDG but also for other tracers, those using population-based input functions, [3], [7], andothers that are based on use of arterialized venous blood sampling, [2]. Alternative techniques are focused on the construction of an imagederived input function, possibly combined with a limited blood sampling and/or simultaneous estimation of both the input function and the kinetic parameters for a limited number of brain regions, [8]-[15]. Noting the risks and discomfort associated with any kind of blood sampling, the PET Neuroimaging Working Group [6] has recommended that, where possible, FDG-PET studies be accomplished through use of a completely image-derived input function. The work in this paper seeks to make further progress toward this end by extending an existing image-derived approach [10].

We propose and validate a new method for the construction of an input function. The specific form is piecewise linear in the early phase of fast uptake of tracer. The piecewise linear approximation is found by fitting with data from an image-derived blood vessel carotid artery region of interest (CA-ROI). It is corrected for partial volume through continuity at a time point common to both windows. The parameters defining the analytic representation of the input function for the later window, including the partial volume coefficient, the rate constants of two representative tissue activity curves defined by cluster analysis, and spillover from blood to tissue, are estimated by a simultaneously estimated (SIME) nonlinear fitting. For fitting within the SIME three blood samples are still required. The approach accounts for partial volume effects and avoids the tissueto-blood spillover.

Details of the method are provided in the following sections. Briefly, the method proceeds as follows. 
Initially, CA-ROIs are identified via a semi-automated procedure, following that presented already in [10]. The method proposed here differs in that only CA-ROI data from very early time are utilized. While the measurement of the input function from image data is sure to be contaminated at all times by partial volume effects caused by the small size of the carotid artery, relative to the image resolution, spillover from tissue to blood from brain regions surrounding the carotid artery can be ignored during the first very short time window (less than one minute duration) because the initial uptake of the tracer by the tissue is essentially insignificant. For the remaining large time interval, spillover from surrounding tissues will contaminate the measurement of the input derived from any region of interest containing the carotid artery. We thus assume that there are two phases of the input function and treat the initial short window differently from the later time measurements. In particular, because the CA-ROI activity curve on the second window is contaminated by spillover, it is replaced in the SIME technique by an analytic representation of the input for this window, the parameters of which are obtained through the SIME by fitting against three intravenous blood samples, which are free of partial volume effects. Continuity of the input is imposed at the point common to both windows. The SIME approach uses data from dominant tissue time activity curves obtained by a clustering technique applied to the volumetric time course data [16].

In the remainder of the paper the rationale for the new method is presented in Section II. All validation steps are performed with the available data from the clinical data set used in [10], briefly described in Section II-A. Results of the clinical study are given in Section III, with a brief discussion of pertinent implementation details presented in Section IV. Finally our conclusions are summarized in Section V.

\section{Rationale And DeVelopment of the}

\section{PRoposed METHOD}

\section{A. Clinical Data}

PET data from 18 healthy subjects who participated in a previous study ( [10]) was used to test the proposed technique. Subjects in this study provided their informed consent, and were studied under guidelines approved by Human-Subjects committees at Banner Good Samaritan Regional Medical Center (Phoenix, AZ) and the Mayo Clinic (Scottsdale, AZ.)

The data used are retrospective and the data collection protocol is described precisely elsewhere [10]. Briefly, PET data was collected using a 951/31 ECAT scanner (Siemens, Knoxville, TN) with the identical scanning protocol for each subject. An initial 20 min transmission scan was acquired for attenuation correction and an intravenous bolus of $10 \mathrm{mCi}$ FDG was administered under resting condition. PET images were reconstructed using a filtered back projection algorithm with Hanning filter of 0.40 cycles per voxel. There are 31 transaxial slices of $128 \times 128$ voxels, each voxel of size $0.18776 \times$ $0.18776 \mathrm{~cm}^{2}$, with a center-to-center slice separation of $3.375 \mathrm{~mm}$ and a $10.8 \mathrm{~cm}$ axial field of view. The final reconstructed PET images have an in-plane resolution of $9.5 \mathrm{~mm}$ full-width at half maximum (FWHM) in the center of the field of view and an axial resolution of 5.0-7.1 mm FWHM. Scanning time durations, given in minutes, for the reconstructed frames are $0.2,8 \times 0.0333$, $2 \times 0.1667,0.2,0.5,2 \times 1,2 \times 1.5,3.5,2 \times 5,10$ and 30.

For the purpose of validation arterial blood samples are required. These were drawn every 5 seconds for the first minute, every 10 seconds for the second minute, every 30 seconds for the next 2 minutes, and then at 
$5,6,8,10,12,15,20,25,30,40,50$ and 60 minutes, yielding a discrete representation of the blood sampled function, denoted by $u_{\mathrm{bs}}\left(t_{j}\right)$, for $j=1, \ldots, 34$.

The implementation of the proposed method only requires three blood samples at approximately $t=10$, 20 and 60 minutes. It has been previously validated, however, that there is good agreement between arterial and venous blood samples, with less than $2.25 \%$ mean difference over these time points, [10]. Thus for the purposes of this retrospective study we used data with existing arterial samples. Moreover, to make sure we have high quality blood data in our experiments we were provided with 4 blood samples. In practice bad samples occur either due to the sampling itself or inadequate counting by the well-counter. The quality of the blood samples is checked for consistency with the expected gradual decrease of tracer in blood and for apparent outliers. Thus, we are guaranteed to have at least 3 high quality blood samples. This approach is consistent with the routine use of the procedure [10].

Practically, for new studies in which arterial sampling is not already intended, the use of venous, rather than arterial samples, as described also in [10] is recommended as a less invasive and less painful method of acquiring the necessary data. The use of late venous samples in order to provide additional information for obtaining an image-derived input function has also been validated in [17], in which venous samples at $30 \mathrm{~min}$ and $60 \mathrm{~min}$ were used.

\section{B. Estimation of the average image-derived blood time} activity curve

Details of the method, which involves summing, slice by slice, the initial time frames up to time 2 minutes in order to emphasize those pixels showing the tracer in blood regions including the CA, are given in [10]. An average blood ROI image-derived input curve, denoted by $v(t)$, is then obtained from all frames in which an individual blood time activity curve can be identified.

\section{Evaluation of models for the input function}

The curves presented in Figures 3, 4 illustrate the two phases of blood activity seen in the carotid artery. In the initial sub window $W_{1}=[0, \tau]$, where typically $\tau<0.6$, the imaged curve $v(t)$ severely underestimates the tracer activity in the blood, due to partial volume effects. The average imaged blood activity curve $v(t)$ on $W_{1}$ provides crucial subject and situation dependent information on the input function, by providing the times at which the tracer reaches the CA-ROI, $\tau_{0}$, and then the time at which it peaks in the blood, $\tau_{p}$. For the window $W_{2}=[\tau, T]$, the impact of spillover on $v(t)$ cannot be negligible. It is thus our premise that these two windows on the input function should be treated differently, using the identifiability of the time points $\tau_{0}, \tau_{p}$, and $\tau$.

1) The input function on $W_{2}$ : To develop an appropriate formulation for modeling the input function on $W_{2}$, we contrast four different input models. The formulation $u_{\text {Phelps }}=A_{1} e^{\lambda_{1}(t-\tau)}+A_{2} e^{\lambda_{2}(t-\tau)}+A_{3} e^{\lambda_{3}(t-\tau)}$,

was first introduced by [2], and later used by [3] as a population-based input function, dependent on the 6 parameters $A_{i}, \lambda_{i}, i=1,2,3$, and here is modified for decay from time $\tau$. This formulation was modified by [1], [17] for use in a SIME method, and is used here with the data shifted relative to $\tau_{0}$ in order to obtain the best possible fit:

$$
\begin{aligned}
u_{\text {Feng }}= & \left(A_{1}\left(t-\tau_{0}\right)-A_{2}-A_{3}\right) e^{\lambda_{1}\left(t-\tau_{0}\right)} \\
& +A_{2} e^{\lambda_{2}\left(t-\tau_{0}\right)}+A_{3} e^{\lambda_{3}\left(t-\tau_{0}\right)} .
\end{aligned}
$$

These two formulations are designed so as to provide a fit for the entire window of time activity $W_{1} \cup W_{2}$, and 


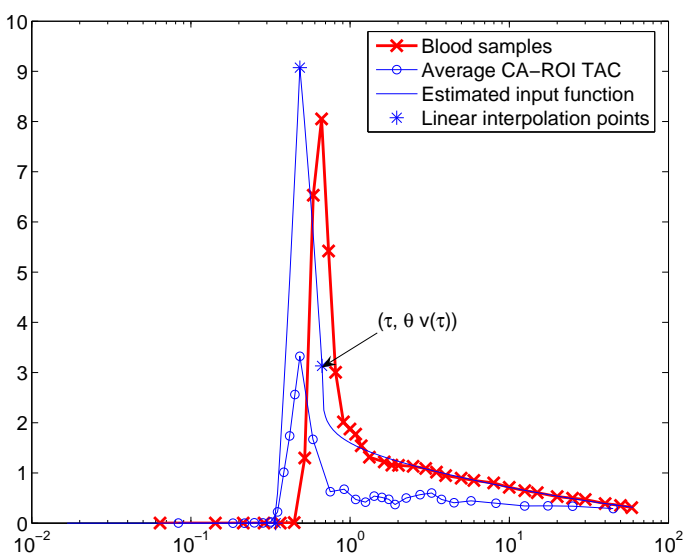

Fig. 1. Comparison of the arterial blood samples (solid line with cross), un-corrected average blood time activity curve (solid line with open circles) and estimated input function solid line with 3 discrete asterisks. Also illustrated, with the 3 asterisks, is the time at which the average blood tracer activity is initiated in the ROI $\tau_{0}$, the time point for the peak value $\tau_{p}$, the point of continuity $\tau$ between windows $W_{1}$ and $W_{2}$, and the three blood samples used for the fit. Time is expressed on logarithm scale hence emphasizing the effects for early time.

may therefore be unnecessarily complicated when fitting just for $W_{2}$. We consider a form with a very simple exponential decay after time $\tau$ :

$$
u_{\operatorname{Exp}}=A e^{\lambda(t-\tau)},
$$

and a more complicated form

$$
u_{\text {new }}=A e^{\lambda(t-\tau)^{\delta}}
$$

To validate (4) as sufficient for fitting arterial data on $W_{2}$ based on just three measured data points and the point of continuity $\tau$, we do a nonlinear fit for each of (1-4) using data points $\tau$, and blood samples for time greater that 10 minutes. In this way we avoid difficulties in picking points between $\tau$ and 10 minutes for which recirculation in the blood input may be evident and may lead to difficulty in the data fitting. Moreover, this comparison is then consistent with our proposed SIME which only uses fitting based on both the value at $\tau$ and the blood samples. We evaluate the sensitivity of the fit relative to the choice of $\tau$ for four different choices of $\tau$, the first four time points in each data set after $\tau_{p}$.

Results of the mean and standard deviation of the root mean square error (RMS) for all formulations, and the different choices of $\tau$, denoted by choice 1 to 4 , over all 18 subjects are reported in Table I. Here, and throughout, the notation $x . y e-z$ indicates the number $x . y \times 10^{-z}$.

We observe that there are too few degrees of freedom in (3) to provide a good fit, while (2) is more sensitive to the choice for $\tau$ than are (1) and (4). As judged by these results (4) performs slightly less well than (1). On the other hand, we note that (4) requires only three points besides $\tau$ whereas (1) requires five, and from the actual fitting illustrated for one subject 5 in Figure 2 it is clear that the fit is better. The fit between time $\tau$ and 10 min underestimates the blood samples for (2) and is very sensitive to the choice of $\tau$ for (1). This does not contradict the results in Table I where the RMS reported does not use the time interval between $\tau$ and $10 \mathrm{~min}$. In summary, (4) is relatively insensitive to the choice for $\tau$ in terms of the fit, and provides a good fit to the entire curve between $\tau$ and the final time point, even when no points between $\tau$ and $10 \mathrm{~min}$ are used. Hence (4) provides an appropriate formulation for use in a nonlinear simultaneous estimation of the input.

2) Determination of time points $\left(\tau_{0}, \tau_{p}, \tau\right)$ : For $W_{1}$, $\tau_{p}$ is determined automatically as the point at which the highest intensity value $v\left(\tau_{p}\right)$ is achieved. While various methods have been proposed for the estimation of the initiation time of activity, $\tau_{0}$, prior to which there is effectively no signal, for example [18], in this work we select $\tau_{0}$ automatically as the point after which $v(t)$ remains greater than $2 \%$ of the peak value $v\left(\tau_{p}\right)$. Although $\tau$ can be chosen in a variety of ways, it was noted in Section II-C. 1 that the fit is relatively insensitive 
TABLE I

THE MEAN AND STANDARD DEVIATION OF THE ROOT MEAN SQUARE ERROR FOR NONLINEAR LEAST SQUARES DATA FITTING FOR FUNCTIONS (1-4) OVER ALL SUBJECTS AND FOR DIFFERENT CHOICES FOR $\tau$.

\begin{tabular}{|c|c|c|c|c|}
\hline & \multicolumn{4}{|c|}{ Analytic Input Function } \\
\hline$\tau$ & $u_{\text {Phelps }}$ & $u_{\text {Feng }}$ & $u_{\text {Exp }}$ & $u_{\text {new }}$ \\
\hline 1 & $1.53 e-2 \pm 8.0 e-3$ & $5.23 e-2 \pm 1.0 e-2$ & $3.65 e-1 \pm 1.1 e-1$ & $1.79 e-2 \pm 1.1 e-2$ \\
2 & $1.44 e-2 \pm 6.2 e-3$ & $1.59 e-2 \pm 7.3 e-3$ & $2.75 e-1 \pm 9.7 e-2$ & $1.69 e-2 \pm 9.9 e-3$ \\
3 & $1.48 e-2 \pm 7.4 e-3$ & $1.51 e-2 \pm 7.7 e-3$ & $2.42 e-1 \pm 8.3 e-2$ & $1.67 e-2 \pm 1.0 e-2$ \\
4 & $1.49 e-2 \pm 6.5 e-3$ & $1.44 e-2 \pm 7.4 e-3$ & $2.50 e-1 \pm 8.7 e-2$ & $1.68 e-2 \pm 1.0 e-2$ \\
\hline
\end{tabular}
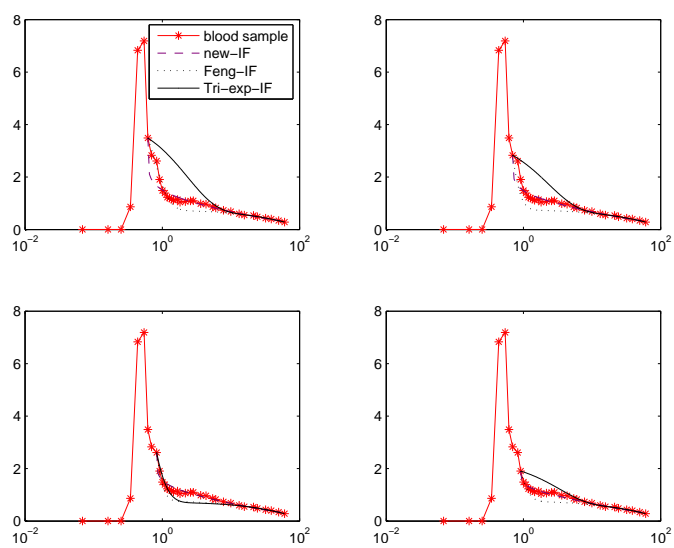

Fig. 2. Fitting results for four choices of $\tau$, illustrated for subject 5 .

to choice of $\tau$. Therefore, we choose $\tau$ automatically as the time point at which $v(t)$ is about $25 \%$ of the peak value $v\left(\tau_{p}\right)$.

3) A parametric model for the input function: Based on the discussion of the preceding paragraphs, we have developed a formulation for the input function, $u_{\mathrm{e}}(t)$.On $W_{1}, v(t)$ is approximated piecewise linearly using the three line segments with nodes $(0,0),\left(\tau_{0}, v\left(\tau_{0}\right)\right)$, $\left(\tau_{p}, v\left(\tau_{p}\right)\right)$ and $(\tau, v(\tau))$. Then, to correct for partial volume, $u_{\mathrm{e}}(t)$ on $W_{1}$ is obtained from the linearized $v(t)$ scaled by a parameter $\theta$ which is inversely proportional to the recovery coefficient of the partial volume coeffi- cient, see Figure 1. On $W_{2}$ we assume decay according to (4) with continuity at $\tau$ imposed. Specifically, in terms of all parameters the estimated form of the input function is given by

$$
u_{\mathrm{e}}(t, \theta, \lambda, \delta)= \begin{cases}0 & t \in\left[0, \tau_{0}\right] \\ \theta v\left(\tau_{p}\right)\left(\frac{\left(t-\tau_{0}\right)}{\left(\tau_{p}-\tau_{0}\right)}\right) & t \in\left(\tau_{0}, \tau_{p}\right] \\ \theta\left(\frac{v(\tau)\left(t-\tau_{p}\right)+v\left(\tau_{p}\right)(\tau-t)}{\left(\tau-\tau_{p}\right)}\right) & t \in\left(\tau_{p}, \tau\right] \\ \theta v(\tau) e^{-\lambda(t-\tau)^{\delta}} & t \in(\tau, \infty) .\end{cases}
$$

In the above model, $v\left(\tau_{p}\right)$ and $v(\tau)$ are known and only the three parameters, $\theta, \lambda$ and $\delta$, need to be determined, and could be obtained simply using the three blood samples for $t>\tau$ along with the value of $v(\tau)$, so as to fit more than 2 parameters. This approach, however, can not compensate or determine the partial volume accurately, which is crucial because of the scaling everywhere due to $\theta$. Instead, while more complicated, simultaneous estimation of the parameters defining the input together with the kinetic parameters of representative tissue TACs provides the necessary additional physical constraints.

\section{Clustering the tissue time activity curves (TTACs)}

The fast clustering method described in detail in [16] is utilized to find representative tissue TACs for the 
whole brain. In this clustering procedure, non-brain, blood vessel and CSF regions are initially excluded by a thresholding step based on the histogram of tracer concentrations at the last time frame. Briefly, the method then uses a hierarchical centroid linkage strategy based on the weighted distance of TACs from the cluster centroid so as to provide average TTACs, $y_{i}^{\mathrm{TAC}}(t)$, for each cluster $i$. The advantage of clustering is that it provides an automated way in which to segment the data into tissue groups of similar tracer activity. In particular, as compared to the original method proposed in [10], there is no need to additionally manually determine the tissue region of interest near the CA-ROI. In practice we automatically cluster to 5 groups and pick from these the two largest for the SIME.

\section{E. Simultaneous estimation of the input function and} cluster kinetic parameters

In the compartmental model [5] for FDG PET, the output $y(t)$ at a given voxel, or for a specific brain tissue ROI, is given analytically by convolution $(\otimes)$ of the instantaneous response function (IRF) with the input $u(t)$

$$
y(t)=u(t) \otimes\left(\frac{K_{1} k_{3}}{k_{2}+k_{3}}+\frac{K_{1} k_{2}}{k_{2}+k_{3}} e^{-\left(k_{2}+k_{3}\right) t}\right) .
$$

Here the IRF is the term within the parentheses and is location dependent through the location dependence of the kinetic parameters. $K_{1}$ is the transport rate from blood to extra-vascular space, $k_{2}$ is the transport rate back from extra-vascular space to blood, and $k_{3}$ is the phosphorylation rate of intra-cellular FDG by hexokinase enzymes to FDG-6-phosphate. In this study the scanning duration of 60 minutes is not long enough to provide a reliable estimate of the dephosphorylation rate of intra-cellular FDG-6-phosphate back to FDG, [4], and which is relatively small compared with other three parameters thus assumed to be 0 . Then, if the input $u(t)$ in (6) is known and spillover effects can be ignored, the parameters defining $y(t)$ for a given location may be estimated using nonlinear (NL) fitting of the measured TAC $y^{\text {TAC }}(t)$ to $y(t)$.

Here, we seek to not only find the parameters of the tissue TACs but also recover the input function. Thus, given representative clustered TTACs for $p$ cluster groups, usually $p=2, y_{i}(t) \approx y_{i}^{\mathrm{TAC}}(t)$, we obtain a weighted nonlinear least squares (WNLS) cost functional in terms of the unknown parameters $\theta$ and $K_{1}^{(i)}, k_{2}^{(i)}, k_{3}^{(i)}$ and $\alpha_{i}$,

$$
\begin{aligned}
& \Phi(\mathbf{x}, \alpha)=\sum_{i=1}^{p} \sum_{j=1}^{n} \\
& w_{j}\left(y_{i}^{\mathrm{TAC}}\left(t_{j}\right)-\alpha_{i} \cdot y_{i}\left(t_{j}\right)-\left(1-\alpha_{i}\right) \cdot u_{\mathrm{e}}\left(t_{j}, \theta\right)\right)^{2} .
\end{aligned}
$$

The weight $w_{j}$ is set to the time duration of each frame, and parameters $\alpha_{i}$ correct for local spillover from blood to tissue, i.e. $\alpha_{i}$ is the tissue fraction in $y_{i}^{T A C}$. To reduce the complexity of the expressions we introduce the notation $\alpha=\left(\alpha_{1}, \ldots, \alpha_{p}\right)$ and $\mathbf{x}=\left[\theta, K_{1}^{(1)}\right.$, $\left.k_{2}^{(1)}, k_{3}^{(1)}, \ldots, K_{1}^{(p)}, k_{2}^{(p)}, k_{3}^{(p)}\right]$. There are in total 11 unknowns which define the SIME.

This expression for the cost functional $\Phi$ hides its actual dependence also on the parameters of the input $u_{\mathrm{e}}(t)$. In particular, the evaluation of $u_{\mathrm{e}}\left(t_{j}, \theta\right)$ occurring in $\Phi(\mathbf{x}, \alpha)$ for a given value $\theta$, requires the nonlinear fit of $u_{\mathrm{e}}$ for $t>\tau$ at points $\left(\tilde{t}_{l}, u_{\mathrm{bs}}\left(\tilde{t}_{l}\right)\right)$ where blood sampled data, $u_{\mathrm{bs}}\left(\tilde{t}_{l}\right), l=1, \ldots, 3$, are obtained for $\tilde{t}_{1} \approx 10, \tilde{t}_{2} \approx 20$ and $\tilde{t}_{3} \approx 60$. The fit is obtained from

$$
\min _{\lambda, \delta} \sum_{i=1}^{3}\left[\theta v(\tau) e^{-\lambda\left(\tilde{t}_{i}-\tau\right)^{\delta}}-u_{\mathrm{bs}}\left(\tilde{t}_{i}\right)\right]^{2} .
$$

In other words, $\lambda$ and $\delta$ are implicitly determined by these three blood samples and are not treated as independent variables for the cost functional $\Phi$. As variable $\theta$ is adjusted for the cost functional $\Phi, \lambda$ and $\delta$ must be 
adjusted accordingly. Thus, the actual total number of independent unknowns in $\Phi(\mathbf{x}, \alpha)$ is 9 .

In order to limit the feasible space of solutions the cost functional $\Phi$ is minimized with respect to its independent variables subject to constraints. The bound constraints on the micro parameters are based on experimental results [4] for both gray and white matter from 13 subjects, but with doubling of upper bounds and halving of lower bounds so as to not provide bounds which may be too conservative. Note that in each case the tissue TACs $y_{i}^{\mathrm{TAC}}$ are automatically shifted to match the first peak of $v(t)$ with that of the TTAC. The constrained global fit performed is

$$
\begin{aligned}
& \min _{\mathbf{x}, \alpha} \Phi(\mathbf{x}, \alpha) \quad \text { subject to } \\
& 1.2 \leq \theta \leq 4 \quad 0.9 \leq \alpha_{i} \leq 1 \\
& 0.015 \leq K_{1}^{(i)} \leq 0.3 \quad 0.024 \leq k_{2}^{(i)} \leq 0.54 \\
& 0.01 \leq k_{3}^{(i)} \leq 0.2 .
\end{aligned}
$$

To solve this minimization we use the Matlab function "fmincon", [19], which uses a sequential quadratic programming (SQP) algorithm. Because of the dependence of $\Phi$ on the blood-sampled data the function call for update of $\Phi$ at each step of the iteration proceeds as follows:

\section{Evaluation of objective function $\Phi(\mathbf{x}, \alpha)$ given current updates for $\alpha, \mathbf{x}$ and $\theta$}

1. Update $u_{\mathrm{e}}\left(t_{j}, \theta\right)$ using (5) and (8), in which blood samples are used to provide the fit in (8) leading to update of $\lambda$ and $\delta$ for the current step.

2. Update the estimates for the cluster TACs using (6) for the current micro parameters, to give $y_{i}\left(t_{j}\right), i=1$ and 2.

3. Update the current value of the objective function $\Phi(\mathbf{x}, \alpha)$ using (7).

\section{RESULTS}

The resulting input functions obtained for the 18 subjects using the described method are compared and validated as follows.

\section{A. Qualitative comparison for the input function}

A comparison of the estimated input function $u_{\mathrm{e}}$ with the blood-sampled input $u_{\mathrm{bs}}$ is illustrated in Figure 3. The upper figure presents the portion within 1.5 minutes, and the middle figure the curves for the whole time duration, 45 minutes, on the logarithmic scale. Because these two curves come from different sources, namely estimation and actual blood samples, there is a time shift between them. In order to provide better comparison $u_{\text {bs }}$ is automatically shifted to match $u_{\mathrm{e}}$, see the lower graph in Figure 3. As expected the tails of both curves are closer because the tail of the estimated curve is obtained by the fit with the blood samples. For all subjects $u_{\mathrm{e}}(t)$ is smoother for later time because it is evaluated using a functional form. Representative comparisons of $u_{\mathrm{e}}$ with shifted $u_{\mathrm{bs}}$ are shown in Figure 4 . The heights of the peaks $u_{\mathrm{e}}\left(\tau_{p}\right)$ match well in all cases, with differences in peak always less than $20 \%$.

\section{B. Estimation of the model parameters}

The estimates obtained for $\theta, \lambda$ and $\delta$ are provided in Table II, along with the average values for each parameter in each case. The spillover from blood to tissue, $1-\alpha_{i}$, for the group TACs is remarkably consistent across all subjects and clusters, and well within the range prescribed by the constraints. On the other hand, partial volume coefficients of the blood ROIs, $1 / \theta$, vary considerably because of the differences in their location (artery or venous), size and shape between subjects. The consistency of the estimate for $\delta$ around the average of 0.248 suggests the robustness of (4) with respect to 

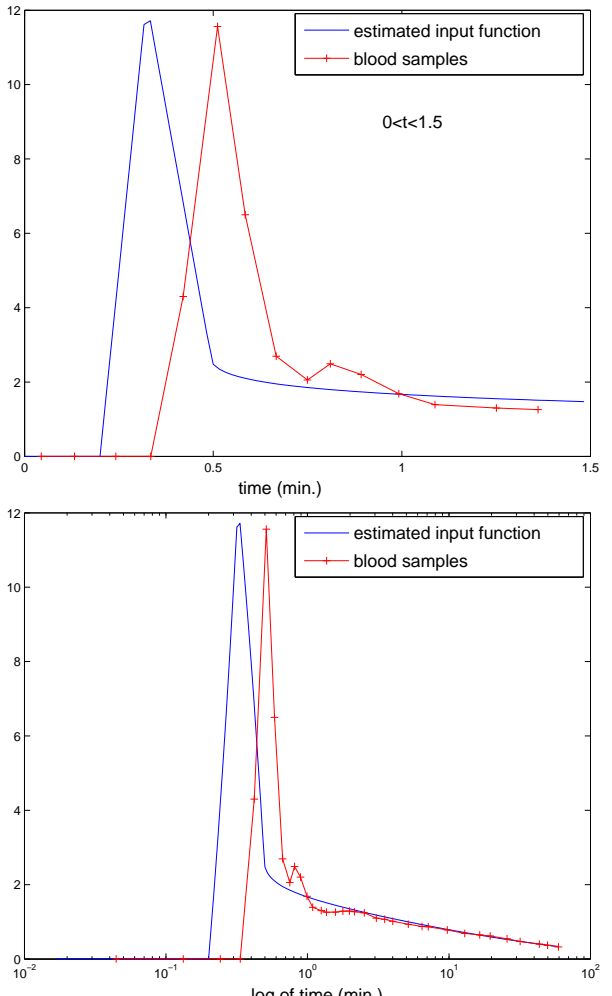

log of time (min.)

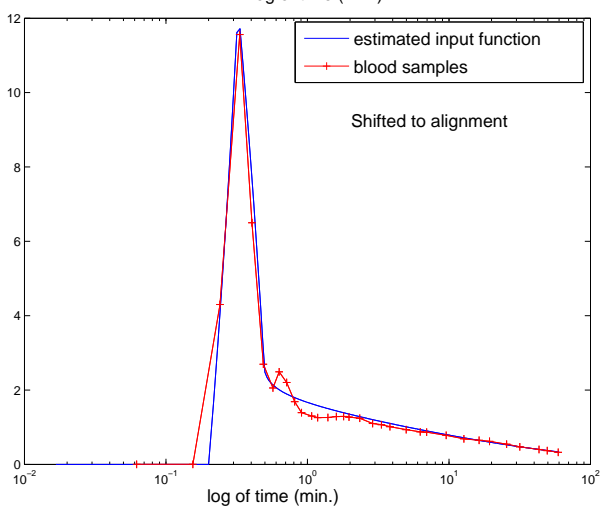

Fig. 3. The recovered input function $u_{\mathrm{e}}(t)$ compared with arterial blood samples. Illustrated are the data focused on the initial window (top), the whole time window given on logarithm scale (middle), and the same data with $u_{\mathrm{e}}(t)$ shifted to account for the time delay relative to the arterial blood samples (bottom).
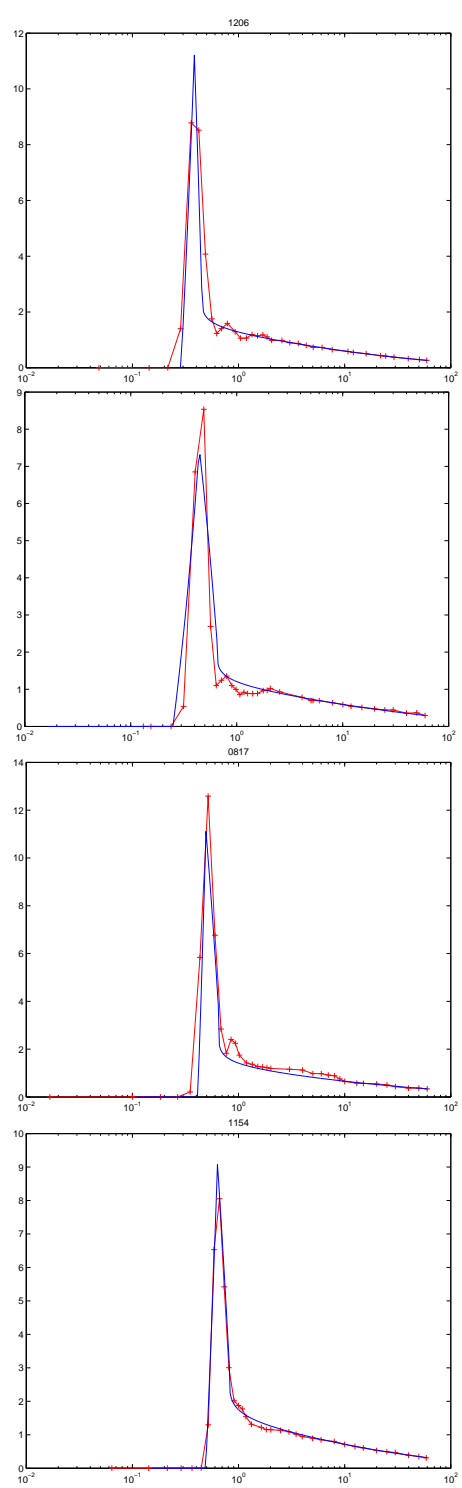

Fig. 4. Comparison of estimated input $u_{\mathrm{e}}$, continuous line, and arterial blood-sampled input $u_{\mathrm{bs}}$, solid line with crosses, for representative subjects, $1,2,3$ and 4 from top to bottom. 
TABLE II

PARAMETERS OBTAINED IN SIME PROCEDURES.

\begin{tabular}{|c|c|c|c|c|c|}
\hline Subject & $\theta$ & $\lambda$ & $\delta$ & $\alpha_{1}$ & $\alpha_{2}$ \\
\hline 1 & 2.049 & 0.923 & 0.233 & 0.930 & 0.931 \\
\hline 2 & 1.975 & 0.805 & 0.226 & 0.923 & 0.927 \\
\hline 3 & 2.843 & 0.691 & 0.241 & 0.935 & 0.935 \\
\hline 4 & 2.730 & 0.872 & 0.237 & 0.958 & 0.957 \\
\hline 5 & 1.790 & 0.518 & 0.318 & 0.912 & 0.915 \\
\hline 6 & 2.294 & 0.828 & 0.265 & 0.938 & 0.932 \\
\hline 7 & 2.287 & 0.350 & 0.364 & 0.928 & 0.929 \\
\hline 8 & 3.190 & 0.797 & 0.257 & 0.935 & 0.934 \\
\hline 9 & 2.927 & 0.919 & 0.231 & 0.953 & 0.968 \\
\hline 10 & 2.813 & 1.554 & 0.153 & 0.918 & 0.927 \\
\hline 11 & 2.651 & 0.521 & 0.342 & 0.933 & 0.932 \\
\hline 12 & 2.246 & 0.628 & 0.247 & 0.930 & 0.925 \\
\hline 13 & 3.543 & 0.781 & 0.277 & 0.941 & 0.941 \\
\hline 14 & 1.908 & 0.937 & 0.212 & 0.941 & 0.940 \\
\hline 15 & 3.781 & 0.792 & 0.257 & 0.942 & 0.940 \\
\hline 16 & 2.822 & 1.152 & 0.167 & 0.935 & 0.935 \\
\hline 17 & 3.070 & 0.924 & 0.214 & 0.951 & 0.944 \\
\hline 18 & 2.996 & 0.842 & 0.222 & 0.947 & 0.924 \\
\hline Average & 2.662 & 0.824 & 0.248 & 0.936 & 0.935 \\
\hline
\end{tabular}

different subjects. Moreover, the upper bound 4.0 for $\theta$ is never attained, so that partial volume coefficients are always greater than $25 \%$. Additional tests, not reported here, in which we used 1 or 3 clusters in the SIME, provide very consistent results.

\section{Quantitative validation with Patlak analysis using the recovered input function}

To provide a comparison with the method presented in [10], data from the entire brain volume are clustered to 50 clusters for each subject, and the recovered input then used in Patlak analysis for each cluster tissue TAC.

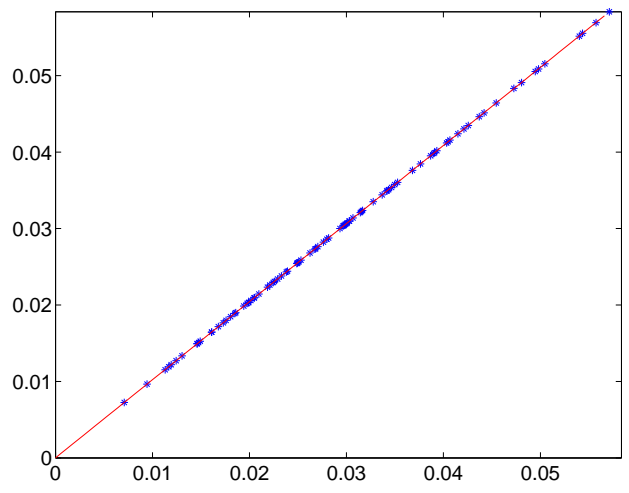

Fig. 5. Regression analysis on $K$ generated by the Patlak method, for blood samples and recovered input function. Slope 1.021, intercept $1.2 e-5$ and correlation coefficient 1.0000 .

Results are compared with those obtained using the blood sampled data, see Table III for all subjects and Figure 5 for subject 5 . The results are in excellent agreement, and better than presented in the original reference, [10].

\section{Quantitative validation via postestimation of kinetic} parameters using the recovered input

To further validate the method, the kinetic parameters were calculated on a cluster by cluster basis not only through the SIME estimated input but also for the measured input from the arterial blood samples. The same Matlab function, "fmincon", was utilized for the solution of the nonlinear minimization with known input function, with the same imposed constraints and applied for the same 50 clusters in each case as used in Section III-C. In each case the output peak is automatically aligned with that of the input and blood to tissue spillover is considered. The estimates of the obtained parameters, $K_{1}, k_{2}, k_{3}$ as well as for the parameter $K=$ $K_{1} k_{3} /\left(k_{2}+k_{3}\right)$ were then compared by linear regression, see Figure 6 in which the regression is shown for subject 
TABLE III

REgRESSION ANALYSis FOR $K$, WHICH IS CALCULATED By THE PATLAK METHOD FOR EACH CLUSTER, USING THE BLOOD SAMPLES AND THE RECOVERED INPUT. MEAN OF $K$ FOR BOTH INPUTS, REGRESSION PAREMETERS, SLOPE, INTERCEPT AND CORRELATION COEFFICIENTS $r$, ARE PRESENTED FOR EACH SUBJECT.

\begin{tabular}{|c|c|c|c|c|c|}
\hline Subject & $\begin{array}{c}\text { mean } K \\
\text { blood input }\end{array}$ & $\begin{array}{c}\text { mean } K \\
\text { estimated input }\end{array}$ & $r$ & slope & intercept \\
\hline 1 & $2.5345 e-2$ & $2.5572 e-2$ & $1.0000 e-0$ & 1.009 & $3.0 e-6$ \\
\hline 2 & $1.8060 e-2$ & $1.8936 e-2$ & $9.9999 e-1$ & 1.046 & $4.0 e-5$ \\
\hline 3 & $1.6389 e-2$ & $1.6030 e-2$ & $9.9999 e-1$ & 0.984 & $-1.0 e-4$ \\
\hline 4 & $1.9380 e-2$ & $1.9669 e-2$ & $9.9998 e-1$ & 1.014 & $1.7 e-5$ \\
\hline 5 & $2.1755 e-2$ & $2.2256 e-2$ & $1.0000 e-0$ & 1.023 & $5.2 e-6$ \\
\hline 6 & $2.2913 e-2$ & $2.2604 e-2$ & $1.0000 e-0$ & 0.986 & $2.2 e-6$ \\
\hline 7 & $1.9948 e-2$ & $1.9675 e-2$ & $1.0000 e-0$ & 0.986 & $-4.5 e-7$ \\
\hline 8 & $1.5685 e-2$ & $1.5922 e-2$ & $9.9999 e-1$ & 1.014 & $1.2 e-5$ \\
\hline 9 & $1.3549 e-2$ & $1.4048 e-2$ & $1.0000 e-0$ & 1.037 & $-3.3 e-8$ \\
\hline 10 & $1.8952 e-2$ & $1.9121 e-2$ & $1.0000 e-0$ & 1.009 & $-5.5 e-6$ \\
\hline 11 & $2.0437 e-2$ & $2.0989 e-2$ & $1.0000 e-0$ & 1.027 & $2.8 e-6$ \\
\hline 12 & $1.5948 e-2$ & $1.6736 e-2$ & $9.9994 e-1$ & 1.052 & $-4.1 e-5$ \\
\hline 13 & $2.5184 e-2$ & $2.5779 e-2$ & $1.0000 e-0$ & 1.023 & $6.7 e-6$ \\
\hline 14 & $2.1261 e-2$ & $2.1666 e-2$ & $1.0000 e-0$ & 1.020 & $-2.0 e-5$ \\
\hline 15 & $2.7067 e-2$ & $2.7237 e-2$ & $1.0000 e-0$ & 1.006 & $6.7 e-6$ \\
\hline 16 & $1.5009 e-2$ & $1.5683 e-2$ & $9.9993 e-1$ & 1.051 & $-9.1 e-5$ \\
\hline 17 & $1.8305 e-2$ & $1.8458 e-2$ & $9.9999 e-1$ & 1.011 & $-4.3 e-5$ \\
\hline 18 & $1.8494 e-2$ & $1.8243 e-2$ & $9.9997 e-1$ & 0.987 & $-1.9 e-5$ \\
\hline
\end{tabular}

5 , Figure 7 in which the regression is shown for a worse set of results for subject 1 and Table IV which lists the slopes, intercepts and correlation coefficients for each subject and each parameter.

Although not all results are presented here, a careful examination of all parameters shows that in no case do any of the parameters reach their bounds, suggesting that the chosen bounds are again appropriate in the context of this analysis.

\section{DISCUSSION}

A. The necessity of SIME for fitting parameters defining the input function

In Section II-C.3 we claimed that the fitting of the proposed model with just the three blood samples for $t>\tau$ used to determine the parameters without using SIME would yield an inaccurate estimate. Here we present a comparison between estimates obtained with and without information from the TACs. Specifically we compare the results in which we directly fit the model 
TABLE IV

REGRESSION FOR THE PARAMETERS OF THE CLUSTERS COMPARING THE BLOOD SAMPLED AND THE RECOVERED INPUT FUNCTIONS. FOR PARAMETERS $k_{1}, k_{2}, k_{3}$ AND $K$, THE CORRESPONDING PARAMETERS ARE, SLOPES $s_{1}, s_{2}, s_{3}$ AND $s_{K}$, INTERCEPTS $b_{1}, b_{2}, b_{3}$ AND $b_{K}$ AND CORRELATION COEFFICIENTS $r_{1}, r_{2}, r_{3}$ AND $r_{K}$.

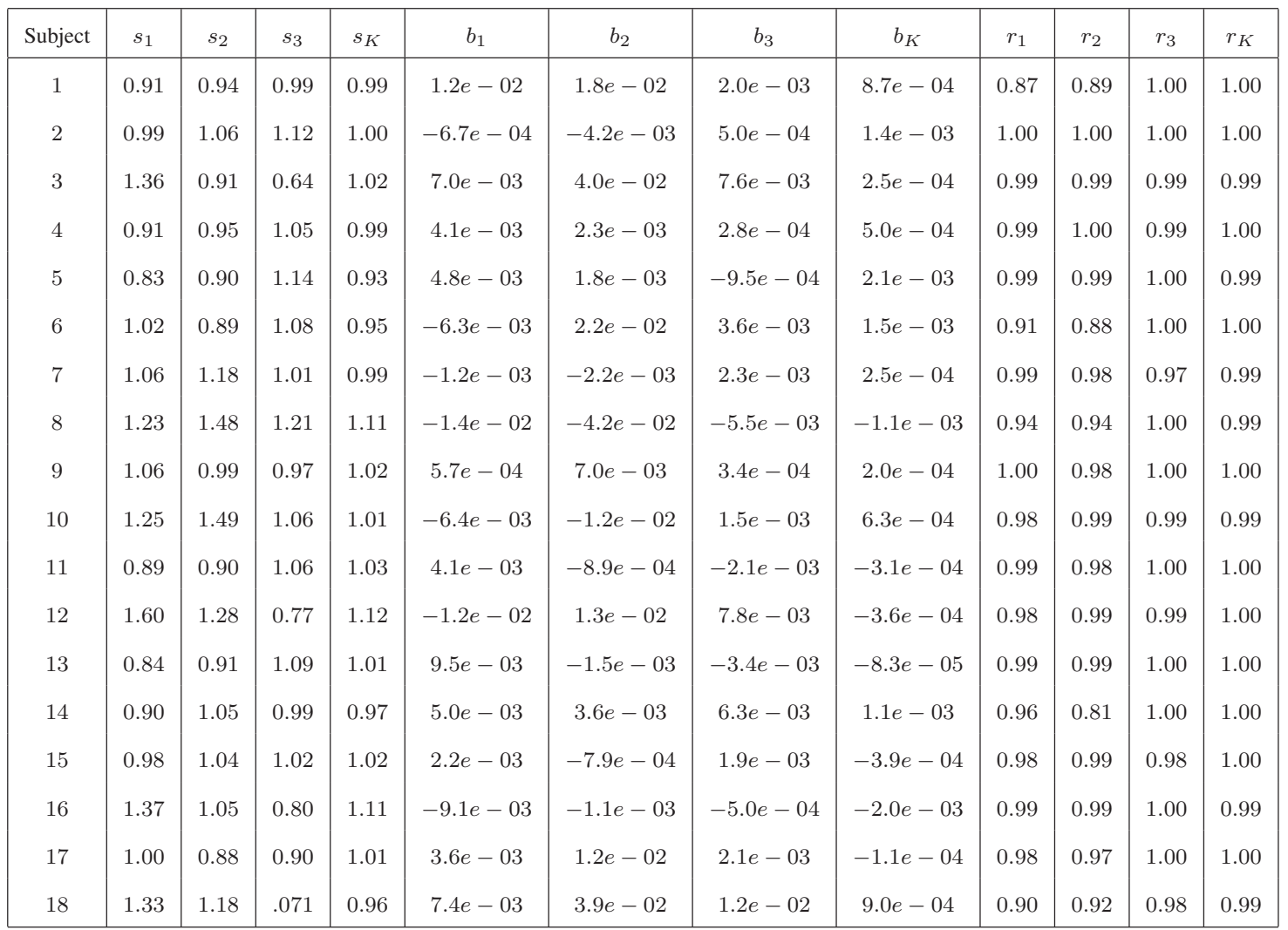

using extrapolation based on data from the three blood samples and in which we simultaneously fit the blood sample data and the tissue TACs. The calculated results for subject 7 are shown in Figure 8. Without information from the tissue TACs the data on the early time window is shifted too high, $\theta=3.909$, because of the inability to correctly estimate the partial volume effects.
B. Time Shift between blood samples and the imagederived blood time activity curve

As we can see from the figures, there is always a time shift between the CA-ROI TAC and the measured blood samples for early time. This then raises a question of whether we need to also include a shift of the data on $W_{1}$ measured from the CA-ROI to match with that on $W_{2}$ which is obtained directly from the three blood samples. We used the arterial blood samples to determine that the actual shift for subject 4 is 10 seconds. The blood samples at approximate times 10, 30 and 60 minutes 

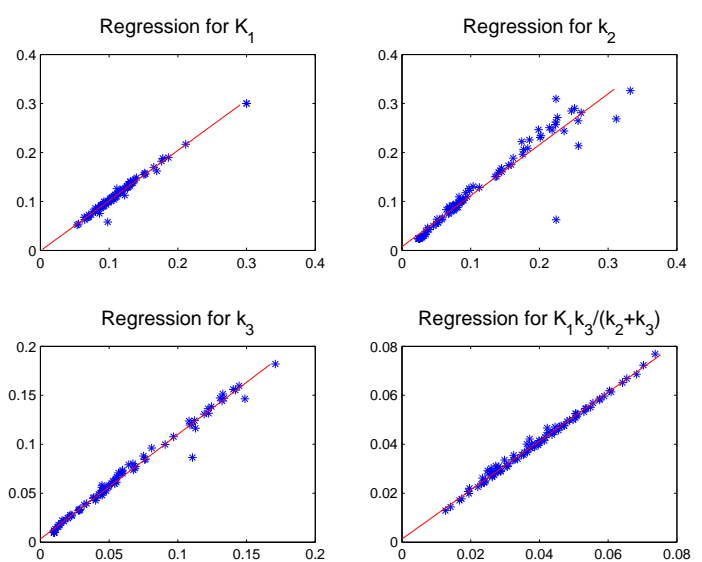

Fig. 6. Comparing the two sets of parameters calculated by the estimated and the arterial blood sampled inputs for subject 5 .
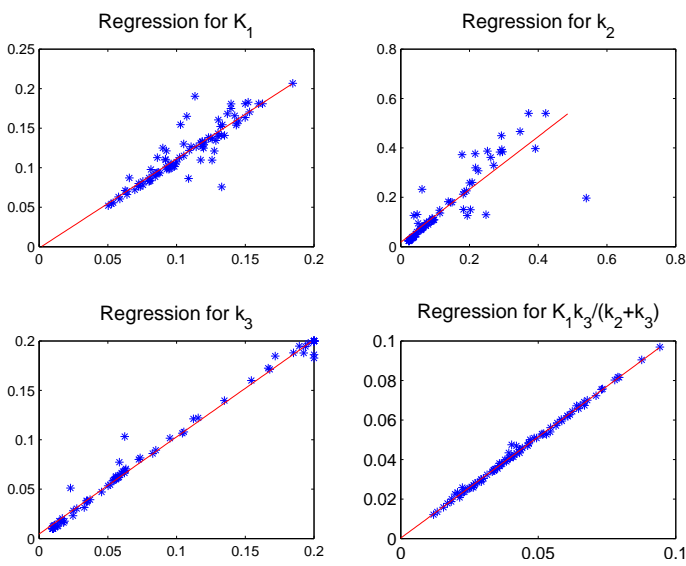

Fig. 7. Comparing the two sets of parameters calculated by the estimated and the arterial blood sampled inputs for subject 1.

are $0.7150,0.4487,0.3130$, resp. Supposing all blood samples for $t>\tau$ are shifted by 10 seconds to the right, the differences after fitting all data by (8), the differences at these same three time points are just $-.004,-.001$, -.0005 , resp. with relative errors less than 0.0056 . Thus the shift of $W_{1}$ relative to $W_{2}$ is negligible. In particular, it has already been demonstrated elsewhere that errors of this magnitude in the input function are insignificant in terms of parametric estimation, [20].

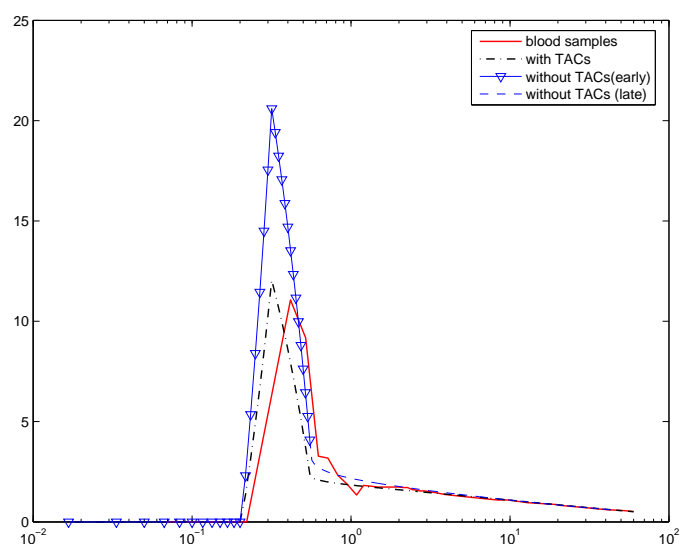

Fig. 8. Comparing the input functions estimated with and without using the tissue TACs in the fitting for subject 7 . Direct fitting of the blood samples without simultaneously incorporating the tissue TACs yields $\theta=3.909$, while with SIME $\theta=2.287$, which yields a much better fit with the baseline blood samples.

C. Advantage of utilizing two windows in defining the input function

The method requires the estimation of $\tau$. This point of continuity between the two windows separates the short window $W_{1}$ on which there is rapid accumulation and fast washout of tracer in the input function, from the window $W_{2}$ on which the tracer concentration in the input decays smoothly. While $\tau$ can be easily defined automatically or manually, its significance is to assure that the most reliable data are used for $W_{1}$ and that $W_{2}$ can be well-modeled by (4). The use of the linear interpolation on $W_{1}$ is a crude simplification, but it is a very good approximation to the real process when $\tau$ is very small. Clearly, it is also possible to use higher order fitting [3] rather than linear interpolation on $W_{1}$ but with $\tau<1 \mathrm{~min}$. the impact will be insignificant. On the other hand, for later time, for which spillover from tissue to blood may contaminate the blood ROI TAC, three samples are utilized to obviate this difficulty. As compared to other image-based input function estimation 
methods, [8], [9], [10], [11], [13] and [12], which use the entire CA-ROI TAC for recovery of the input function, the proposed method is more robust to noise in identification of the input function at later times. it avoids the need to superimpose the CA ROI on later time frames which can compound the difficulty with a poor signal to noise ratio due to potential subject movement over the time course of the study.

\section{The SIME Procedure}

In order to provide reasonable solutions in a cost effective manner, it is important that bounds on the parameters of the nonlinear minimization are provided. Increasing the number of unknowns defining the input function quickly raises the complexity of the nonlinear optimization through the dependence of the fit also on the parameters of the input function. While different weights in the NLS might lead to differences, experiments with the square root of the time duration, or the unweighted case, gave inferior results. The cost function could also be set up so that the two parameters $\lambda$ and $\delta$ can be estimated simultaneously, through the penalized cost functional

$$
\begin{aligned}
& \Phi(\mathbf{x}, \alpha, \delta, \lambda)=\sum_{i=1}^{m} \sum_{j=1}^{n} w_{j}\left(y_{i}^{\mathrm{TAC}}\left(t_{j}\right)\right. \\
& \left.-\alpha_{i} \cdot y_{i}\left(t_{j}\right)-\left(1-\alpha_{i}\right) \cdot u_{\mathrm{e}}\left(t_{j}, \theta, \lambda, \delta\right)\right)^{2} \\
& +\gamma \sum_{i=1}^{p}\left(\theta v(\tau) e^{-\lambda\left(\tilde{t}_{i}-\tau\right)^{\delta}}-u_{\mathrm{bs}}\left(\tilde{t}_{i}\right)\right)^{2},
\end{aligned}
$$

in which $u_{\mathrm{e}}\left(t_{j}, \theta, \delta, \lambda\right)$ has four independent variables and would be evaluated only using (5), and without the fit (8). Here, however, we do not propose this approach because we suppose that the increased complexity of the minimization requires more computations and will not necessarily significantly improve accuracy. Moreover, the internal step (8) of obtaining $\lambda$ and $\delta$ from the three blood samples at each iteration, is very fast because of the simplicity of that data fit.

\section{Conclusions}

A reliable semi-automated alternative for input function estimation which uses 3 blood samples has been presented and validated by FDG PET analysis for 18 healthy subjects. Macro parameter $K$, and micro parameters, $K_{1}$, $k_{2}$ and $k_{3}$ are well estimated as compared to the use of traditional arterial blood sampling. Because of the generality of the approach, and its relative simplicity for implementation, the proposed method should also work for other tracers. The Matlab-based implementation of the tool is available at web site http://math.asu.edu/ hongbin

For future work it is planned to use pharmokinetic analysis of the tracer input for estimation of the input peak value, such as to possibly separate the estimation of the input from that of the kinetics of the data. It is also of interest to validate the method for use with small animal studies (microPET), [21].

\section{REFERENCES}

[1] D. Feng, S.-C. Huang, and X. Wang, "Models for computer simulation studies of input functions for tracer kinetic modeling with positron emission tomography," Int. J. Biomed. Comput., vol. 32, pp. 95-110, 1993.

[2] M. E. Phelps, S.-C. Huang, E. J. Hoffman, C. E. Selin, and D. Kuhl, "Tomographic measurement of local cerebral glucose metabolic rate in man with $\left({ }^{18} \mathrm{~F}\right)$ fluorodeoxyglucose: Validation of method," Ann. Neurol., vol. 6, pp. 371-388, 1979.

[3] S. Eberl, A. R. Anayat, R. R. Fulton, P. K. Hooper, and M. J. Fulham, "Evaluation of two population based input functions for quantitative neurological FDG PET studies," Eur. J. Nucl. Med., vol. 24, pp. 299-304, 1997.

[4] S.-C. Huang, M. E. Phelps, E. J. Hoffman, K. Sideris, C. J. Selin, and D. E. Kuhl, "Noninvasive determination of local cerebral metabolic rate of glucose in man," Am. J. Physiol., vol. 238, no. E, pp. 69-82, 1980.

[5] L. Sokoloff, M. Reivich, C. Kennedy, M. H. D. Rosiers, C. S. Patlack, K. D. Pettigrew, M. Sakurada, and M. Shinohara, "The $\left[{ }^{14} \mathrm{C}\right]$ deoxyglucose method for the measurement of local cerebral glucose metabolism: theory procedures and normal values in 
the conscious and anesthetized albino rat," J. Neurochem., vol. 28, pp. 897-916, 1977.

[6] PET-Working Group. (2005) PET Working Group: NIH/NIA Neuroimaging Initiative. [Online]. Available: http: //www.nia.nih.gov/ResearchInformation/ExtramuralPrograms/ Neuroscie\%nceOfAging/Summary+ $\ \%$ E2 $\backslash \% 80 \backslash \% 93+\mathrm{PET}+$ Working+Group.htm

[7] S. Takikawa, V. Dhawan, P. Spetsieris, W. Robeson, T. Chaly, R. Dahl, D. Margouleff, and D. Eidelberg, "Noninvasive quantitative fluorodeoxyglucose PET studies with an estimated input function derived from a population-based arterial blood curve," Radiology, vol. 188, pp. 131-136, 1993.

[8] J.-E. Litton, "Input function in PET brain-studies using MRI defined arteries," J. Comp. Ass. Tom., vol. 21, no. 6, pp. 907-909, 1997.

[9] M. Liptrot, K. H. Adams, L. Martiny, L. H. Pinborg, M. N. Lonsdale, N. V. Olsen, S. Holm, C. Svarer, and G. M. Knudsen, "Cluster analysis in kinetic modelling of the brain: A noninvasive alternative to arterial sampling," Neuroimage, vol. 21, no. 2, pp. 483-493, 2004.

[10] K. Chen, D. Bandy, E. Reiman, S.-C. Huang, M. Lawson, D. Feng, L.-S. Yun, and A. Palant, "Noninvasive quantification of the cerebral metabolic rate for glucose using positron emission tomography, ${ }^{18} \mathrm{~F}$-fluorodeoxyglucose, the Patlak method, and an image-derived input function," J. Cereb. Blood Flow Metab., vol. 18, pp. 716-723, 1998.

[11] L. M. Wahl, M. C. Asselin, and C. Nahmias, "Regions of interest in the venous sinuses as input functions for quantitative PET," $J$. Nucl. Med., vol. 40, no. 10, pp. 1666-1675, 1999.

[12] M. C. Asselin, V. J. Cunningham, S. Amano, R. Gunn, and C. Nahmias, "Parametrically defined cerebral blood vessels as non-invasive blood input functions for brain PET studies," Phys. Med. Biol., vol. 49, no. 6, pp. 1033-54, 2004.

[13] S. M. Sanabria-Bohorquez, A. Maes, P. Dupont, G. Bormans, T. de Groot, A. Coimbra, W. Eng, T. Laethem, I. D. Lepeleire, J. Gambale, J. M. Vega, and H. D. Burns, "Image-derived input function for $\left[{ }^{11} \mathrm{C}\right]$ flumazenil kinetic analysis in human brain," Mol. Imag. Biol., vol. 5, no. 2, pp. 72-78, 2003.

[14] D. G. Feng, K.-P. Wong, C.-M. Wu, and W.-C. Siu, "A technique for extracting physiological parameters and the required input function simultaneously from PET image measurements: Theory and simulation study," IEEE Trans. Inform. Technol. Biomed., vol. 1, no. 4, pp. 243-254, 1997.

[15] A. Nelson, F. Miraldi, R. F. Muzic Jr., G. P. Leisure, and W. E. Semple, "Noninvasive arterial monitor for quantitative oxygen15-water blood flow studies," J. Nuclear Medicine, vol. 34, no. 6, pp. 1000-1006, 1993.
[16] H. Guo, R. Renaut, K. Chen, and E. Reiman, "Clustering huge data sets for parametric PET imaging," Biosystems, vol. 71, no. 1-2, pp. 81-92, 2003.

[17] K.-P. Wong, D. Feng, S. R. Meikle, and M. J. Fulham, "Simultaneous estimation of physiological parameters and the input function - In vivo PET data," IEEE Trans. Inform. Technol. Biomed., vol. 5, no. 1, pp. 67-76, 2001.

[18] M. E. Raichle, W. R. Martin, P. Herscovitch, M. A. Mintun, and J. Markham, "Brain blood flow measured with intravenous ${ }^{15} \mathrm{O}-$ $\mathrm{H}_{2} \mathrm{O}$ II. Implementation and validation," J. Nucl. Med, vol. 24, pp. 790-798, 1983.

[19] The Mathworks, Optimization Toolbox User's Guide, March 2005.

[20] K. Chen, S.-C. Huang, and D.-C. Yu, "The effects of mesurement errors in the plasma radioactivity curve on parameter estimation in positron emission tomography," Phy. Med. Biol., vol. 36, no. 9, pp. 1183-1200, 1991.

[21] S.-C. Huang, H.-M. Wu, K. Shoghi-Jadid, D. B. Stout, A. Chatziioannou, H. R. Schelbert, and J. R. Barrio, "Investigation of a new input function validation approach for dynamic mouse microPET studies," Molecular Imaging and Biology, vol. 6, no. 1, pp. 3446, 2004.

Hongbin Guo was born in China in 1964. He received the Ph.D. degree from the Department of Mathematics, Fudan University, in 2000.

$\mathrm{He}$ is currently Assistant Research Professor with the Department of Mathematics and Statistics at Arizona State University, which he joined in 2000 as a postdoctoral research associate. His research interests include numerical linear algebra and its applications in medical imaging, signal processing and bioinformatics.

Rosemary A. Renaut was born in Kingswood, United Kingdom, in 1958. She received the B. Sc.. degree in Mathematics from the University of Durham, England, in 1980, Part III of the Applied and Mathematical Physics Tripos in 1981, and the Ph.D. degree in Mathematics in 1985 from the University of Cambridge, England. 
She is a Full Professor in the Department of Mathematics and Statistics at Arizona State University, which she joined in 1987 as an Assistant Professor. She has held several visiting positions, including at the ETH Zurich, Switzerland, and the Technical Universities of Kaiserslautern and Munich in Germany. Her current research interests include numerical methods for linear algebra and optimization for applications using methods such as total least squares for signal restoration, in diverse areas of medical imaging, seismic data analysis, and other relevant fields. She is a Chartered Mathematician and Fellow of the Institute of the Mathematics and its Applications of the United Kingdom.

Kewei Chen was born in Hebei Province, China, in 1957. He received the B. Sc.. degree in Mathematics from Beijing Normal University, China, in 1980, the M. Sc. in Biomathematics from the same institute in 1985, and the Ph.D. degree in Biomathematics in 1993 from the University of California, Los Angeles, USA.

$\mathrm{He}$ is the director of the Computational Image Analysis Program at Banner PET Center, and adjunct Professor in the Department of Mathematics and Statistics at Arizona State University. He has held several other visiting positions, including at Beijing Normal University, Beijing, China, and Dalian University of Technology, Dalian, China. His current research interests primarily fall into the neuroimaging study of Alzheimer's disease and the general methodology studies in the neuroimaging field including PET, MRI and fMRI. 